\title{
Driving Factors for the Utilisation of Healthcare Services by People with Osteoarthritis: Results from A Nationwide Population-Based study
}

Daniela Costa ( $\sim$ dcosta.ft@gmail.com )

Universidade NOVA de Lisboa https://orcid.org/0000-0001-8469-6356

Ana M Rodrigues

NOVA Medical School, Universidade NOVA de Lisboa

Eduardo B Cruz

Escola Superior de Saúde - Instituto Politécnico de Setúbal

Helena Canhão

NOVA Medical School - Universidade NOVA de Lisboa

Jaime da Cunha Branco

NOVA Medical School - Universidade NOVA de Lisboa

\section{Carla Nunes}

Universidade Nova de Lisboa Escola Nacional de Saude Publica

\section{Research article}

Keywords: Delivery of health care, Osteoarthritis, Socioeconomic factors, Health services, Andersen's behavioural model of healthcare utilisation

Posted Date: March 2nd, 2021

DOl: https://doi.org/10.21203/rs.3.rs-279474/v1

License: (9) This work is licensed under a Creative Commons Attribution 4.0 International License. Read Full License 


\section{Abstract}

Background: Worldwide, the current management of knee osteoarthritis appears heterogeneous, high-cost and often not based on current best evidence. The absence of epidemiological data regarding the utilisation of healthcare services may conceal the need for improvements in the management of osteoarthritis. The aim of this study is to explore the profiles of healthcare services utilisation by people with knee osteoarthritis, and to analyse their determinants, according to Andersen's behavioural model. Methods: We analysed a sample of 978 participants diagnosed with knee osteoarthritis from the population-based study EpiReumaPt. Data was collected with a structured interview, and the diagnosis of knee osteoarthritis was validated by a rheumatologist team. With the Two-step Cluster procedure, we primarily identified different profiles of healthcare utilisation according to the services most used by patients with knee osteoarthritis. Secondly, we analysed the determinants of each profile, using multinomial logistic regression, according to the predisposing characteristics, enabling factors and need variables.

Results: In our sample, a high proportion of participants are overweight or obese $(82,6 \%, \mathrm{n}=748)$ and physically inactive $(20,6 \%, n=201)$ and a small proportion had physiotherapy management $(14,4 \%$, $\mathrm{n}=141)$. We identified three profiles of healthcare utilisation: "HighUsers"; "GPUsers"; "LowUsers". "HighUsers" represents more than $35 \%$ of the sample, and are also the participants with higher utilisation of medical appointments. "GPUsers" represent the participants with higher utilisation of general practitioner appointments. Within these profiles, age and geographic location - indicated as predisposing characteristics; employment status and healthcare insurance - as enabling factors; number of comorbidities, physical function, health-related quality of life, anxiety and physical exercise - as need variables, showed associations $(p<0,05)$ with the higher utilisation of healthcare services profiles.

Conclusions: Healthcare utilisation by people with knee osteoarthritis is not driven only by clinical needs. The predisposing characteristics and enabling factors associated with healthcare utilisation reveal inequities in the access to healthcare and variability in the management of people with knee osteoarthritis. Research and implementation of whole-system strategies to improve equity in the access and quality of care are paramount in order to diminish the impact of osteoarthritis at individual-, societaland economic-level.

\section{Introduction}

Osteoarthritis (OA) is a leading cause of disability worldwide, responsible for 9.6 million years-lived with disability, of which $85 \%$ are attributable to the knee joint (1). The direct costs of knee OA represent $1-2.5 \%$ of the GDP of high-income countries, mainly accounted by Total Knee Replacement surgery (TKR) costs. Moreover, the indirect costs can surpass the direct costs, mainly due to work loss or early retirement (2). In Portugal, $12,4 \%$ of the adult population have knee OA (3) and, in 2013, the indirect costs represented $0.4 \%$ of the GDP (4). Portugal has an ageing population, where $80 \%$ of the elderly people are overweight 
and $75 \%$ of the adult population is physically inactive (5). This data suggests a progressive and future increase in the prevalence and burden of knee OA, in the same way as in other countries (6).

People with knee OA suffer from chronic pain, fatigue, sleep problems, disability, impaired quality of life and mental health, this limits their participation in social, community and occupational activities $(3,7)$. The management of this condition requires integrated multi-disciplinary interventions during the progression of the disease to reduce pain, modify the risk factors and improve function, as there is no known cure for OA (7).

Exercise, maintenance of a healthy body weight, education and self-management strategies are recommended as first-line and core interventions during the disease progression. Pharmacological modalities can help with the symptoms control. TKR should only be considered if the core interventions have failed and, if HRQoL is significantly impaired in selected patients $(8,9)$, due mainly to the rates of surgical complications and adverse events, associated mortality and low levels of satisfaction with the outcomes (10).

However, data from several countries suggests that the current care for knee $\mathrm{OA}$ is heterogeneous and discordant with the quality standards (11). Medication for pain relief is often the first line treatment prescribed by general practitioners (GP's) (12), less than $50 \%$ of patients are referred to physiotherapy or weight management programs and referrals to the orthopaedic surgeon is often inadequate (11). Portugal is the country with highest TKR growth rate among OECD countries, where the incidence rate increased by $20 \%$ between 2005 and 2011 for patients both above and below 65 years old (13).

International data has shown that overall healthcare utilisation and related costs are significantly higher in patients with knee OA than in the matched non-OA population, even when adjusted for the number of comorbidities (14). Moreover, the variability in healthcare utilisation can be driven by determinants other than clinical factors, like sex, education level, income, insurance coverage, perceived needs, area of residence and socio-economic status (15).

According to the Andersen's behavioural model, the utilisation of healthcare services is influenced by individual determinants, that can be classified in three domains: 1) predisposing characteristics demographic variables that influence people to use healthcare services; 2 ) enabling factors - socioeconomic related factors that promote the utilisation; 3 ) need variables - clinically related causes that foster people to seek healthcare. In an equitable system, the interventions received should be driven by the patient's clinical needs (16).

The Portuguese National Health Service (NHS) is a universal coverage, tax-financed system and GP's act as a gatekeeper to other health services. Additionally, private health insurance and, health insurance schemes that cover particular professions, facilitate the access to the private healthcare sector (17).

Currently, there is no published data about healthcare utilisation by people with knee OA in Portugal, and the literature with national datasets is scarce. Due to the complexity of this condition, the identification of 
different profiles of healthcare services utilisation and its determinants is critical to identify needs for improvement at individual and system level and, to develop interventional strategies to mitigate these needs. The aim of this study is to explore different profiles of healthcare services utilisation by people with knee OA and to analyse its determinants, according to Andersen's behavioural model. Secondarily, we aim to describe the overall healthcare services used by people with knee OA.

\section{Material And Methods}

\section{Data source}

This study analyses the EpireumaPt project database, a national cross-sectional population-based study with a representative sample of the Portuguese population. EpiReumaPt aimed to develop a comprehensive understanding of the burden of Rheumatic and Musculoskeletal Diseases (RMD's) in Portugal. As described in detail elsewhere (18), the EpiReumaPt study recruitment used a three-phase approach, over the period September 2011 to December 2013. The sample of EpiReumaPt study was recruited from a random selection of private households in Portuguese Mainland and Islands (Madeira and Azores), and was stratified according to the administrative territorial units [(NUTS II) (Norte, Centro, Lisboa and Vale do Tejo, Alentejo, Algarve, Açores Islands (Azores) and Madeira Islands (Madeira)], and the size of the population within each locality $(<2000 ; 2000-9999 ; 10,000-19,999 ; 20,000-99,999$; and $\geq 100,000$ inhabitants, respectively). In each household, an individual $\geq 18$ years old with permanent residence and the most recently celebrated birthday was selected to be a participant in the study. Each selected household was visited, with no previous contact, up to three times, if no candidate participant was present during the first visit. In the long run, 28,502 households were contacted, 8041 individuals refused to participate in the study, and 10,661 were included. The EpiReumaPt population was similar to the Portuguese population (CENSUS 2011) in age strata, gender, and NUTII distribution (18).

In the first phase of the study, the participants completed a face-to-face interview to collect health-related information, which also screened for RMD's, by a team of non-medical healthcare professionals trained for purpose. The interviews were conducted with a Computer Assisted Personal Interview (CAPI) system. An individual was considered to have a positive screening if the subject mentioned a previously known $\mathrm{RMD}$, if any of the algorithms in the screening questionnaires was positive, or if the subject reported muscle, vertebral or peripheral joint pain in the previous 4 weeks.

The overall performance of the screening algorithm was evaluated (the gold standard was considered the final diagnosis after revision - phase 3 ) and the overall sensitivity of the screening questionnaire

for RMD's was $98 \%$, with a specificity of $22 \%$. The positive predictive value was $85 \%$ and the negative predictive value was $71 \%(18)$.

The participants who screened positive for at least one RMD $(n=7451)$, as well as approximately $20 \%$ ( $n$ $=701$ ) of participants with negative screening for RMD's, were invited for a second phase, that consisted 
of a clinical appointment with a Rheumatologist. Of these, 4275 did not attend the clinical appointment. Therefore, at the end of phase 2 there were 3877 clinical observations: 3198 received the validation of RMD'S and 679 did not have an RMD diagnosis. The clinical assessments were performed at the Primary Care Centre of the participants neighbourhood, with a mobile van, fully equipped, to perform imaging and laboratory tests, supported by a multidisciplinary team with a rheumatologist, and X-Ray technician, a nurse, a staff coordinator and a driver. The clinical appointments consisted of a structured evaluation, laboratory and imaging exams, if needed, to establish the diagnosis and evaluate disease-related information. The rheumatologists involved were blind to the prior health-related data. In the third-phase, three experienced rheumatologists reviewed all the data and confirmed the diagnosis - Fig. 1 (18).

When data was insufficient to fulfil international classification criteria for each RMD, a meeting of 5 rheumatologists took place in order to reach an agreement on the final diagnosis. When doubts persisted the opinion of the rheumatologist that performed the clinical assessment (second phase) prevailed. Diagnostic agreement between the 3 reviewers was $98.3 \%$ with a Cohen's $\mathrm{K}$ coefficient of $10.87(95 \% \mathrm{Cl}$ from 0.83 to 0.91 ). A total of 981 participants had a validated diagnosis of knee $O A(18)$.

Please place here "Figure 1. Flowchart of study design" - Legends in the end of the manuscript

\section{Study population}

This study includes the participants of EpiReumaPt with knee OA diagnosis, validated in the second phase of EpireumaPt, according to the American College of Rheumatology criteria: knee pain with at least three of the following clinical findings: age $>50$ years, morning stiffness $<30$ minutes duration, crepitus in active motion, tenderness of the bone margins of the joint, bony enlargement noted on examination, and lack of palpable warmth of the synovium (19).

\section{Outcomes}

The healthcare services utilisation data is the outcome of interest, collected in the first phase of the study. Participants were asked if they had attended any medical appointments, undergone hospitalisations, surgery, psychology and physiotherapy consultations and, to indicate the number of appointments in the previous 12 months. The number of General Practitioner (GP) appointments was categorised in "no appointments", "1-2 appointments" and "3 or more appointments". The reason for hospitalisation was asked. Joint surgery was considered, although the presented variable does not distinguish between joint replacement surgery or other joint surgery. During the database cleaning, data from participants who had more than 60 medical appointments or 180 physiotherapy sessions were considered as measurement errors and were excluded $(n=3)$.

\section{Determinants}

The determinant variables were collected during the first phase of EpiReumaPt, and are presented according to Andersen's model, previously described. To assure a better clinical interpretation, some variables were subjected to categorical transformation. 


\section{Predisposing characteristics}

Predisposing characteristics included: age, sex, geographic location, according to NUTS II territorial units, previously described. Madeira and Azores were merged in the analysis as Islands region. Marital status was merged in having a partner (married or consensual union)/do not have a partner (single, widowed or divorced).

\section{Enabling factors}

These factors included: work status, firstly presented as employed (full or part time), retired, unemployed, incapable of working due to rheumatic disease and others (domestic worker, students, live with revenues) and then categorised as employed and non-employed (unemployed, retired, incapable of working due to rheumatic diseases and others); have or do not have healthcare insurance, additional to NHS coverage; number of years of schooling, that was categorised as having $<4$ and $\geq 4$ years of schooling, representing the attendance (or not) of at least the first stage of primary education.

\section{Need variables}

Need variables included the number of self-reported chronic comorbidities: high blood pressure, high cholesterol, cardiac disease, diabetes mellitus, chronic lung disease, problems in the digestive tract, renal colic, neurological disease, allergies, mental or psychiatric illness, cancer, thyroid and parathyroid problems, hypogonadism, hyperuricemia. The presence of other rheumatic diseases (excluding knee OA), diagnosed by the rheumatologists' team, was added. Body mass index $\left(\mathrm{kg} / \mathrm{m}^{2}\right)$ was calculated with selfreported height and weight, and categorised as underweight $\left(\leq 18.49 \mathrm{~kg} / \mathrm{m}^{2}\right)$, healthy weight $(\geq 18.5$ and $\left.\leq 24.99 \mathrm{~kg} / \mathrm{m}^{2}\right)$, overweight $\left(\geq 25\right.$ and $\left.\leq 29.99 \mathrm{~kg} / \mathrm{m}^{2}\right)$ and obese $\left(\geq 30 \mathrm{~kg} / \mathrm{m}^{2}\right)$. Lifestyle variables such as alcohol intake and smoking habits (both categorised as never, occasionally and daily) were noted, as well as past habits of smoking. Regular physical exercise/sports habits were also asked (yes/no). Healthrelated quality of life (HRQoL) was assessed using EuroQol, with 5 dimensions and 3 levels (EQ-5D-3L) (20). The index score ranges from 1 , which represents full health, and zero or below that corresponds to death or states worse than death. Anxiety and depression symptoms were evaluated using the Hospital Anxiety and Depression Scale (HADS) for subscales of depression (HADS-D) and anxiety (HADS-A). Both fall into a range from 0 to 21, where higher values represent greater symptoms of anxiety or depression (21). Physical function was measured based on the Health Assessment Questionnaire (HAQ). Where total scores lying between zero, indicating no functional impairment, and 3 indicating complete impairment (22). The use of regular medication and number of medicines was also collected.

\section{Statistical analysis}

All statistical analyses were performed using SPSS 24 for MacOS (IBM Corp., Armonk, NY, USA).

In the first stage, using descriptive statistic methods, we explored the health services most used by participants with knee OA. With the results of this analysis and the knowledge of the literature previously published in this field (23), we included in the Two Step Cluster (TSC) procedure four variables: GP 
appointments categorised (no appointments, 1-2 appointments and $\geq 3$ appointments in the previous 12 months), orthopaedic surgeon and physiotherapy appointments (yes/no), and hospitalisation (yes/no), in the past 12 months. The categorisation of the variable "GP appointments" was made according to the median value of the distribution of this variable in the sample $(x=3.00)$.

The TSC procedure is a hybrid approach that uses a distance measure to separate groups, and an agglomerative hierarchical clustering based on best fit to choose the optimal subgroup model. In this procedure, we used the Schwarz's Bayesian information criterion (BIC) as a statistical measure of best fit to determine the number of clusters, the log-likelihood as distance measure and the average silhouette coefficient (ASC) as the silhouette measure of cluster cohesion and separation. We accepted the cluster solution considering the highest ratio of distance measure. Evidence shows that TSC is one of the most reliable procedures in terms of the number of subgroups detected, classification probability of individuals to subgroups and reproducibility of findings on clinical data (24).

We used descriptive statistics and non-parametric tests for independent samples (Kruskal-Wallis for continuous variables and chi-squared test for categorical variables, $p<0.05$ ) to describe and compare the determinants and health utilisation in the entire sample and between clusters.

In addition, through a sensitive analysis to assure the robustness of our results, we also explored the association between the determinant variables and the cluster membership. First, we conducted a univariate analysis to select the variables for inclusion in the multinomial logistic regression model, with a significance level of 0.2 , to avoid early exclusion of potential important variables. Then, in the multinomial regression procedure, we performed a stepwise hierarchical analysis according to the domains of Andersen's model in three steps: 1) inclusion of predisposing characteristics in the model and removal of non-significant variables; 2 ) inclusion of enabling factors in the previous model and removal of non-significant enabling factors; 3 ) inclusion of need variables in the previous model and removal of non-significant need variables, resulting in the final model. Odds-Ratio (OR) was estimated for each variable with $95 \%$ confidence interval $(\mathrm{Cl})$. Participants with missing data were automatically excluded from this analysis. This model was adjusted for gender and age therefore, we forced the entry of these variables in all steps.

We evaluated the discriminative capacity of each model in each of the three steps calculating a binomial area under the receiver operating curve (AUC), to analyse the proportion of increment in the discriminative capacity in each step. The binomial AUC was calculated using the estimated classification probability for a given cluster, regarding the reference cluster. The discriminative capacity was considered weak if AUC was between 0.5-0.69; acceptable if between 0.7-0.79 and good if above 0.80 . We also analysed the variance of the multinomial model using the McFadden Pseudo- $\mathrm{R}^{2}$ in each step (25).

\section{Ethics}

EpireumaPt study was approved by the Ethics Committee of NOVA Medical School and by the Portuguese Data Protection Authority (Comissão Nacional de Proteção de Dados). Written informed consent was 
obtained from all participants, in accordance with the Declaration of Helsinki, as described elsewhere (18).

\section{Results}

\section{Profiles of healthcare utilisation}

Among the 978 participants diagnosed with knee OA included in the analysis, we found three different profiles of healthcare services utilisation with the TSC procedure, based on the healthcare services most used - Table 1. We named the clusters according to the attendance to orthopaedic surgeon appointments, physiotherapy sessions, number of GP appointments and hospitalisation. 
Table 1

Healthcare utilisation of total sample and Clusters

\begin{tabular}{|c|c|c|c|c|c|}
\hline & $\begin{array}{l}\text { Total }^{\mathrm{f}} \\
\mathrm{n}(\%) \\
978 \\
(100)\end{array}$ & $\begin{array}{l}\text { HighUsers } \\
\mathrm{n}(\%) \\
343 \\
(35.07)\end{array}$ & $\begin{array}{l}\text { GPUsers } \\
\mathrm{n}(\%) \\
272 \\
(27.80)\end{array}$ & $\begin{array}{l}\text { LowUsers } \\
\mathrm{n}(\%) \\
363 \\
(37.11)\end{array}$ & p-value \\
\hline \multicolumn{6}{|l|}{ Cluster Variables ${ }^{a}$} \\
\hline \multicolumn{6}{|l|}{ GP Appointments } \\
\hline 0 & $\begin{array}{l}126 \\
(12.9)\end{array}$ & $30(8.7)$ & $0(0)$ & $96(26.4)$ & \multirow[t]{3}{*}{$\begin{array}{l}< \\
0.001^{d}\end{array}$} \\
\hline $1-2$ & $\begin{array}{l}379 \\
(38.8)\end{array}$ & $\begin{array}{l}112 \\
(32.7)\end{array}$ & $0(0)$ & $267(73.6)$ & \\
\hline$\geq 3$ & $\begin{array}{l}473 \\
(48.4)\end{array}$ & $\begin{array}{l}201 \\
(58.6)\end{array}$ & $272(100)$ & $0(0)$ & \\
\hline Physiotherapy ${ }^{b}$ & $\begin{array}{l}141 \\
(14.4)\end{array}$ & $\begin{array}{l}141 \\
(41.1)\end{array}$ & $0(0)$ & $0(0)$ & $\begin{array}{l}< \\
0.001^{d}\end{array}$ \\
\hline Orthopaedic Surgeon ${ }^{b}$ & $\begin{array}{l}192 \\
(19.6)\end{array}$ & $\begin{array}{l}192 \\
(56.0)\end{array}$ & $0(0)$ & $0(0)$ & $\begin{array}{l}<.001^{d} \\
0\end{array}$ \\
\hline Hospitalisation $^{b}$ & $\begin{array}{l}112 \\
(11.5)\end{array}$ & $\begin{array}{l}112 \\
(32.7)\end{array}$ & $0(0)$ & $0(0)$ & $\begin{array}{l}< \\
0.001^{d}\end{array}$ \\
\hline \multicolumn{6}{|l|}{ Healthcare Use } \\
\hline GP appointments, mean \pm SD & $\begin{array}{l}3.14 \pm \\
3.32\end{array}$ & $\begin{array}{l}3.38 \pm \\
3.38\end{array}$ & $\begin{array}{l}4.00 \pm \\
1.17\end{array}$ & $\begin{array}{l}1.27 \pm \\
0.83\end{array}$ & $\begin{array}{l}< \\
0.001^{\mathrm{e}}\end{array}$ \\
\hline \multicolumn{6}{|l|}{ Other medical appointments ${ }^{b}$} \\
\hline Rheumatology & $56(5.7)$ & $29(8.5)$ & $12(4.4)$ & $15(4.1)$ & $0.026^{d}$ \\
\hline Physiatry & $43(4.4)$ & $39(11.4)$ & $4(1.5)$ & $0(0)$ & $\begin{array}{l}< \\
0.001^{d}\end{array}$ \\
\hline Cardiology & $\begin{array}{l}136 \\
(13.9)\end{array}$ & $72(21.0)$ & $25(9.2)$ & $39(10.7)$ & $\begin{array}{l}<.001^{d} \\
0\end{array}$ \\
\hline Neurology & $50(5.1)$ & $24(7.0)$ & $17(6.3)$ & $9(2.5)$ & $0.015^{d}$ \\
\hline Internal medicine & $77(7.9)$ & $10.8(37)$ & $24(8.8)$ & $16(4.4)$ & $0.006^{d}$ \\
\hline Surgery & $71(7.3)$ & $55(5.6)$ & $8(2.9)$ & $8(2.2)$ & $\begin{array}{l}< \\
0.001^{d}\end{array}$ \\
\hline
\end{tabular}




\begin{tabular}{|c|c|c|c|c|c|}
\hline & $\begin{array}{l}\text { Totalf }^{f} \\
\mathrm{n}(\%)\end{array}$ & $\begin{array}{l}\text { HighUsers } \\
\mathrm{n}(\%)\end{array}$ & $\begin{array}{l}\text { GPUsers } \\
\mathrm{n}(\%)\end{array}$ & $\begin{array}{l}\text { LowUsers } \\
\mathrm{n}(\%)\end{array}$ & p-value \\
\hline & $\begin{array}{l}978 \\
(100)\end{array}$ & $\begin{array}{l}343 \\
(35.07)\end{array}$ & $\begin{array}{l}272 \\
(27.80)\end{array}$ & $\begin{array}{l}363 \\
(37.11)\end{array}$ & \\
\hline Psychiatry & $45(4.6)$ & $19(5.5)$ & $15(5.5)$ & $11(3.0)$ & $0.197^{d}$ \\
\hline $\begin{array}{l}\text { Other medical appointments }{ }^{c} \text {, mean } \\
\pm \text { SD }\end{array}$ & $\begin{array}{l}2.41 \pm \\
4.35\end{array}$ & $\begin{array}{l}4.43 \pm \\
6.65\end{array}$ & $\begin{array}{l}3.82 \pm \\
4.75\end{array}$ & $1.82 \pm$ & $\begin{array}{l}<.001^{\mathrm{e}} \\
0.00\end{array}$ \\
\hline Joint Surgery ${ }^{b}$ & $20(2.3)$ & $20(8.0)$ & $0(0)$ & $0(0)$ & $<.001^{d}$ \\
\hline Psychology ${ }^{b}$ & $13(1.3)$ & $5(1.5)$ & $1(0.4)$ & $7(1.9)$ & $0.228^{d}$ \\
\hline Technical aids ${ }^{\mathrm{b}}$ & $18(1.8)$ & $17(5.0)$ & $1(0.4)$ & $0(0)$ & $<.001^{d}$ \\
\hline \multicolumn{6}{|c|}{$\begin{array}{l}\text { Categorical variables are presented as } \mathrm{n}(\%) \text {; continuous variables are presented as mean } \pm \text { standard } \\
\text { deviation }\end{array}$} \\
\hline \multicolumn{6}{|l|}{ GP, General Practitioner. } \\
\hline \multicolumn{6}{|c|}{ 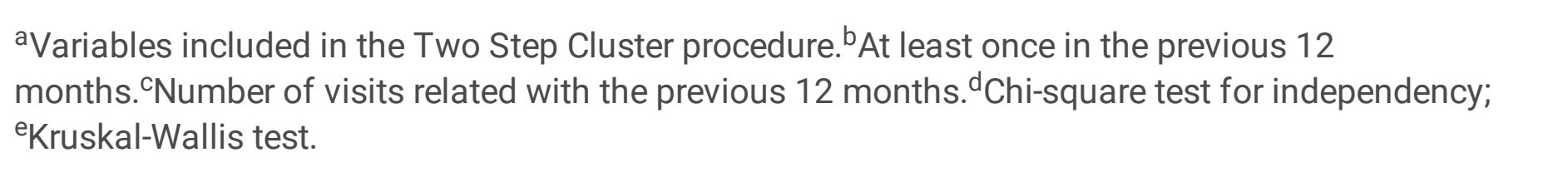 } \\
\hline \multicolumn{6}{|c|}{$\begin{array}{l}\text { f Sample Size is not constant due to the following: Joint Surgery }(n=885) \text { and Technical aids }(n= \\
973) \text {. }\end{array}$} \\
\hline
\end{tabular}

1. "High Healthcare Users" (HighUsers): included all participants from the sample who had at least one appointment in the previous 12 months with the orthopaedic surgeon, who had physiotherapy and who had hospitalisation. In this cluster, the distribution of participants among the three categories of GP appointments was heterogeneous. The participants included in the HighUsers cluster represent $35.07 \%$ of the sample.

2. "GP users" (GPUsers): included only participants who had 3 or more GP appointments in the last 12 months and no use of the other services. Participants included in the GPUsers cluster represent $27.8 \%$ of the sample.

3. "Low healthcare users" (LowUsers): included participants who had less than 2 appointments with the GP in the previous 12 months, and no use of the other healthcare services. Participants included in LowUsers cluster represent $37.11 \%$ of the sample

This cluster solution presents an ASC of 0.6 , which shows a good model fit, and the ratio of distance measures was 1.706. 
Regarding the total sample, $87.2 \%$ of the participants reported at least one GP visit, $14.4 \%$ were enrolled in physiotherapy, $19.6 \%$ had visited the orthopaedic surgeon and $11.50 \%$ were hospitalised in the last 12 months. HighUsers represent the participants with higher number of medical appointments $(4.43 \pm 6.65$, $\mathrm{p}<0.001$ ) among the majority of medical specialities. GPUsers, despite the higher number of GP appointments $(4.00 \pm 1.17, p<0.001)$, were the ones with higher regular medication consumption $(1.12 \pm$ $3.86, p<0.001)$.

\section{Characteristics of the sample and clusters}

Women represent $73 \%$ of the sample, the mean age of participants was $65.34( \pm 11.30)$ years old, 247 (25.3\%) participants have less than four years of education, and only $15 \%$ of participants were employed. The majority of the participants are overweight $(41.8 \%)$ or obese $(40.8 \%)$ and only $20.6 \%$ report doing regular physical exercise. Distributions across clusters were statistically different $(p<0.05)$ for the majority of predisposing characteristics, enabling factors and need variables - Table 2 . 
Table 2

Predisposing characteristics, enabling factors and need variables distribution of total sample and clusters

\begin{tabular}{|c|c|c|c|c|c|c|}
\hline & Determinants & $\begin{array}{l}\text { Total }^{\mathrm{a}} \\
\mathrm{n}(\%)\end{array}$ & $\begin{array}{l}\text { HighUsers } \\
\mathrm{n}(\%)\end{array}$ & $\begin{array}{l}\text { GPUsers } \\
\mathrm{n}(\%)\end{array}$ & $\begin{array}{l}\text { LowUsers } \\
\mathrm{n}(\%)\end{array}$ & $\begin{array}{l}p- \\
\text { value }\end{array}$ \\
\hline & & $\begin{array}{l}978 \\
(100)\end{array}$ & $\begin{array}{l}343 \\
(35.07)\end{array}$ & $\begin{array}{l}272 \\
(27.80)\end{array}$ & $\begin{array}{l}363 \\
(37.11)\end{array}$ & \\
\hline PREDISPOSING & Age & $\begin{array}{l}65.34 \pm \\
11.30\end{array}$ & $\begin{array}{l}65.18 \pm \\
10.51\end{array}$ & $\begin{array}{l}66.47 \pm \\
10.35\end{array}$ & $\begin{array}{l}64.74 \pm \\
12.02\end{array}$ & $0.081^{b}$ \\
\hline & Female gender & $\begin{array}{l}714 \\
(73)\end{array}$ & $\begin{array}{l}259 \\
(75.5)\end{array}$ & $\begin{array}{l}209 \\
(76.8)\end{array}$ & $\begin{array}{l}246 \\
(67.8)\end{array}$ & $0.017^{c}$ \\
\hline & Geographic Location & & & & & \\
\hline & North & $\begin{array}{l}255 \\
(29.3)\end{array}$ & $\begin{array}{l}105 \\
(34.0)\end{array}$ & $\begin{array}{l}60 \\
(28.6)\end{array}$ & $81(25.2)$ & $<.001$ \\
\hline & Center & $\begin{array}{l}243 \\
(27.9)\end{array}$ & $85(27.2)$ & $\begin{array}{l}87 \\
(36.1)\end{array}$ & $72(22.4)$ & \\
\hline & Islands & $\begin{array}{l}127 \\
(14.6)\end{array}$ & $32(10.4)$ & $21(8.7)$ & $74(23.1)$ & \\
\hline & South & $84(9.6)$ & $29(9.4)$ & $\begin{array}{l}26 \\
(10.8)\end{array}$ & $29(9.0)$ & \\
\hline & Lisbon & $\begin{array}{l}162 \\
(18.6)\end{array}$ & $59(19.1)$ & $\begin{array}{l}38 \\
(15.8)\end{array}$ & $65(20.2)$ & \\
\hline & $\begin{array}{l}\text { Married or } \\
\text { consensual union }\end{array}$ & $\begin{array}{l}619 \\
(63.3)\end{array}$ & $\begin{array}{l}222 \\
(64.7)\end{array}$ & $\begin{array}{l}165 \\
(60.7)\end{array}$ & $\begin{array}{l}232 \\
(63.9)\end{array}$ & $\begin{array}{l}0.556 \\
c\end{array}$ \\
\hline ENABLING & Years of Education & $\begin{array}{l}4.96 \pm \\
3.32\end{array}$ & $\begin{array}{l}5.05 \pm \\
3.36\end{array}$ & $\begin{array}{l}4.36 \pm \\
3.18\end{array}$ & $\begin{array}{l}5.32 \pm \\
3.32\end{array}$ & $\begin{array}{l}< \\
0.001^{\mathrm{b}}\end{array}$ \\
\hline & $\begin{array}{l}<4 \text { years of } \\
\text { Education }\end{array}$ & $\begin{array}{l}247 \\
(25.3)\end{array}$ & $79(23.1)$ & $\begin{array}{l}98 \\
(36.0)\end{array}$ & 70 (19.3) & $\begin{array}{l}< \\
0.001^{c}\end{array}$ \\
\hline & Employment status & & & & & $\begin{array}{l}0.006 \\
c\end{array}$ \\
\hline & $\begin{array}{l}\text { Employed (part-time } \\
\text { OR full-time) }\end{array}$ & $\begin{array}{l}145 \\
(16.6)\end{array}$ & $42(13.5)$ & $\begin{array}{l}31 \\
(12.9)\end{array}$ & $72(22.9)$ & \\
\hline & Retired & $\begin{array}{l}634 \\
(72.4)\end{array}$ & $\begin{array}{l}222 \\
(71.4)\end{array}$ & $\begin{array}{l}185 \\
(76.7)\end{array}$ & $\begin{array}{l}228 \\
(70.2)\end{array}$ & \\
\hline & Unemployed & $68(7.8)$ & $31(10.0)$ & $18(7.5)$ & $19(5.8)$ & \\
\hline & Temporarily disabled & $17(1.9)$ & $11(3.5)$ & $4(1.7)$ & $2(0.6)$ & \\
\hline & Others ${ }^{d}$ & $12(1.4)$ & $5(1.6)$ & $3(1.3)$ & $4(1.2)$ & \\
\hline
\end{tabular}




\begin{tabular}{|c|c|c|c|c|c|c|}
\hline & Determinants & $\begin{array}{l}\text { Total } \\
\text { n (\%) }\end{array}$ & $\begin{array}{l}\text { HighUsers } \\
\text { n (\%) }\end{array}$ & $\begin{array}{l}\text { GPUsers } \\
\text { n (\%) }\end{array}$ & $\begin{array}{l}\text { LowUsers } \\
\mathrm{n}(\%)\end{array}$ & $\begin{array}{l}p- \\
\text { value }\end{array}$ \\
\hline & & $\begin{array}{l}978 \\
(100)\end{array}$ & $\begin{array}{l}343 \\
(35.07)\end{array}$ & $\begin{array}{l}272 \\
(27.80)\end{array}$ & $\begin{array}{l}363 \\
(37.11)\end{array}$ & \\
\hline & $\begin{array}{l}\text { NHS as only health } \\
\text { system }\end{array}$ & $\begin{array}{l}763 \\
(78.0)\end{array}$ & $\begin{array}{l}236 \\
(74.9)\end{array}$ & $\begin{array}{l}230 \\
(79.3)\end{array}$ & $\begin{array}{l}297 \\
(79.6)\end{array}$ & $0.003^{c}$ \\
\hline \multirow[t]{14}{*}{ NEED } & $\begin{array}{l}\text { Physical function } \\
\text { (HAQ) }\end{array}$ & $\begin{array}{l}0.76 \pm \\
0.69\end{array}$ & $\begin{array}{l}0.88 \pm \\
0.66\end{array}$ & $\begin{array}{l}0.77 \pm \\
0.67\end{array}$ & $\begin{array}{l}0.47 \pm \\
0.59\end{array}$ & $\begin{array}{l}< \\
0.001^{b}\end{array}$ \\
\hline & HRQoL (EQ-5D-3L) & $\begin{array}{l}0.62 \pm \\
0.27\end{array}$ & $\begin{array}{l}0.57 \pm \\
0.27\end{array}$ & $\begin{array}{l}0.61 \pm \\
0.26\end{array}$ & $\begin{array}{l}0.73 \pm \\
0.25\end{array}$ & $\begin{array}{l}< \\
0.001^{\mathrm{b}}\end{array}$ \\
\hline & BMI $\left(\mathrm{kg} / \mathrm{m}^{2}\right)$ & $\begin{array}{l}29.54 \pm \\
5.06\end{array}$ & $\begin{array}{l}29.71 \pm \\
4.77\end{array}$ & $\begin{array}{l}29.69 \pm \\
5.14\end{array}$ & $\begin{array}{l}29.27 \pm \\
4.95\end{array}$ & $0.366^{\mathrm{b}}$ \\
\hline & $\begin{array}{l}\text { Underweight }(<18.5 \\
\left.\mathrm{kg} / \mathrm{m}^{2}\right)\end{array}$ & $2(0.2)$ & $0(0)$ & $0(0)$ & $2(0,6)$ & \multirow[t]{4}{*}{$0.311^{\mathrm{c}}$} \\
\hline & $\begin{array}{l}\text { Normal weight (18.5- } \\
\left.24.99 \mathrm{~kg} / \mathrm{m}^{2}\right)\end{array}$ & $\begin{array}{l}156 \\
(17.2)\end{array}$ & 59 (15.2) & $\begin{array}{l}40 \\
(16.1)\end{array}$ & $67(19.9)$ & \\
\hline & $\begin{array}{l}\text { Overweight (25-29.99 } \\
\left.\mathrm{kg} / \mathrm{m}^{2}\right)\end{array}$ & $\begin{array}{l}379 \\
(41.8)\end{array}$ & $\begin{array}{l}135 \\
(41.8)\end{array}$ & $\begin{array}{l}110 \\
(44.4)\end{array}$ & $\begin{array}{l}134 \\
(39.9)\end{array}$ & \\
\hline & Obese $\left(\geq 30 \mathrm{~kg} / \mathrm{m}^{2}\right)$ & $\begin{array}{l}370 \\
(40.8)\end{array}$ & $\begin{array}{l}139 \\
(43.0)\end{array}$ & $\begin{array}{l}98 \\
(39.5)\end{array}$ & $\begin{array}{l}133 \\
(39.6)\end{array}$ & \\
\hline & Anxiety (HADS-A) & $\begin{array}{l}6.86 \pm \\
4.15\end{array}$ & $\begin{array}{l}7.08 \pm \\
4.20\end{array}$ & $\begin{array}{l}7.54 \pm \\
4.23\end{array}$ & $\begin{array}{l}5.85 \pm \\
3.94\end{array}$ & $\begin{array}{l}< \\
0.001^{b}\end{array}$ \\
\hline & Depression (HADS-D) & $\begin{array}{l}6.09 \pm \\
4.30\end{array}$ & $\begin{array}{l}5.74 \pm \\
3.96\end{array}$ & $\begin{array}{l}6.59 \pm \\
4.21\end{array}$ & $\begin{array}{l}4.94 \pm \\
3.82\end{array}$ & $0.001^{b}$ \\
\hline & $\begin{array}{l}\text { Number of } \\
\text { Comorbidities }\end{array}$ & $\begin{array}{l}4.47 \pm \\
2.44\end{array}$ & $\begin{array}{l}4.48 \pm \\
2.42\end{array}$ & $\begin{array}{l}5.11 \pm \\
2.54\end{array}$ & $\begin{array}{l}3.65 \pm \\
2.19\end{array}$ & $<.001^{b}$ \\
\hline & Daily alcohol intake & $\begin{array}{l}199 \\
(20.4)\end{array}$ & $70(20.5)$ & $\begin{array}{l}53 \\
(19.5)\end{array}$ & $76(20.9)$ & $0.077^{c}$ \\
\hline & Active smoker & $71(7.3)$ & $25(7.3)$ & $16(5.9)$ & $30(8.3)$ & $0.519^{c}$ \\
\hline & Ex-smoker & $\begin{array}{l}182 \\
(17.9)\end{array}$ & $59(18.6)$ & $\begin{array}{l}38 \\
(14.8)\end{array}$ & $65(19.5)$ & $0.312^{c}$ \\
\hline & $\begin{array}{l}\text { Regular physical } \\
\text { activity }\end{array}$ & $\begin{array}{l}201 \\
(20.6)\end{array}$ & $58(17.0)$ & $\begin{array}{l}41 \\
(15.1)\end{array}$ & $\begin{array}{l}102 \\
(28.1)\end{array}$ & $\begin{array}{l}< \\
0.001^{c}\end{array}$ \\
\hline
\end{tabular}




\begin{tabular}{|c|c|c|c|c|c|}
\hline Determinants & $\begin{array}{l}\text { Total }^{\mathrm{a}} \\
\mathrm{n}(\%) \\
978 \\
(100)\end{array}$ & $\begin{array}{l}\text { HighUsers } \\
\text { n (\%) } \\
343 \\
(35.07)\end{array}$ & $\begin{array}{l}\text { GPUsers } \\
\text { n (\%) } \\
272 \\
(27.80)\end{array}$ & $\begin{array}{l}\text { LowUsers } \\
\text { n (\%) } \\
363 \\
(37.11)\end{array}$ & $\begin{array}{l}\text { p- } \\
\text { value }\end{array}$ \\
\hline \multicolumn{6}{|c|}{$\begin{array}{l}\text { Categorical variables are presented as } n(\%) \text {; continuous variables are presented as mean } \pm \text { standard } \\
\text { deviation }\end{array}$} \\
\hline \multicolumn{6}{|c|}{$\begin{array}{l}\text { NHS, National Health System; GP, General Practitioner; HRQoL, Health Related Quality of Life; EQ-5D- } \\
\text { 3L, EuroQol with five dimensions and three levels; HAQ, Health Assessment Questionnaire; HADS-A, } \\
\text { Hospital Anxiety and Depression Scale - Anxiety subscale; HADS-D, Hospital Anxiety and Depression } \\
\text { Scale - Depression subscale. }\end{array}$} \\
\hline \multicolumn{6}{|c|}{ asample size is not constant due to the following: } \\
\hline \multicolumn{6}{|c|}{$\begin{array}{l}\text { Geographic location }(n=871) \text {, Years of education }(n=977) \text {, Employment status }(n=876), \text { EQ-5D-3L } \\
\text { score }(n=965) \text {, Alcohol intake }(n=977), \text { BMI }(n=971) \text {, Regular physical activity }(n=977)\end{array}$} \\
\hline \multicolumn{6}{|l|}{${ }^{\mathrm{b}}$ Kusskall-Wallis test } \\
\hline \multicolumn{6}{|l|}{${ }^{c}$ Chi-squared test } \\
\hline${ }^{\mathrm{d}}$ This category includes particip & tuc & omestic $n$ & & & \\
\hline
\end{tabular}

Please place here "Table 2. Predisposing characteristics, enabling factors and need variables distribution of total sample and clusters" (presented in the end of manuscript)

\section{Determinants of Cluster Membership}

After the univariate analysis (Supplementtary file), variables at $<0.2$ significance level were considered for the multinomial logistic regression model. The reference category was LowUsers cluster. Due to missing data, $146(14,93 \%)$ participants were excluded from this analysis, but the proportion of excluded participants was similar between clusters - Table 3. 
Table 3

Final Multinomial Regression Model

HighUsers vs. LowUsers

GPUsers vs. LowUsers

\begin{tabular}{|c|c|c|c|c|c|c|c|}
\hline $\begin{array}{l}\text { Variables } \\
\text { added }\end{array}$ & Determinants & OR & $\mathrm{Cl}$ & $\begin{array}{l}\mathrm{p}- \\
\text { value }\end{array}$ & OR & $\mathrm{Cl}$ & $\begin{array}{l}\mathrm{p} \text { - } \\
\text { value }\end{array}$ \\
\hline \multirow{7}{*}{$\begin{array}{l}\text { Predisposing } \\
\text { Characteristics }\end{array}$} & Age & 0.96 & $\begin{array}{l}0.95- \\
0.99\end{array}$ & 0.001 & 0.99 & $\begin{array}{l}0.96- \\
1.01\end{array}$ & 0.172 \\
\hline & Male gender ${ }^{\mathrm{a}}$ & 1.05 & $\begin{array}{l}0.69- \\
1.58\end{array}$ & 0.826 & 1.12 & $\begin{array}{l}0.72- \\
1.76\end{array}$ & 0.613 \\
\hline & Geographic Location ${ }^{b}$ & & & & & & \\
\hline & North & 1.545 & $\begin{array}{l}0.93- \\
2.56\end{array}$ & 0.091 & 1.23 & $\begin{array}{l}0.70- \\
2.15\end{array}$ & 0.475 \\
\hline & Center & 1.50 & $\begin{array}{l}0.89- \\
2.51\end{array}$ & 0.130 & 2.11 & $\begin{array}{l}1.21- \\
3.68\end{array}$ & 0.008 \\
\hline & Islands & 0.43 & $\begin{array}{l}0.24- \\
0.77\end{array}$ & 0.005 & 0.42 & $\begin{array}{l}0.21- \\
0.83\end{array}$ & 0.013 \\
\hline & South & 1.49 & $\begin{array}{l}0.76- \\
2.94\end{array}$ & 0.250 & 1.82 & $\begin{array}{l}0.89- \\
3.75\end{array}$ & 0.102 \\
\hline Step 1 & AUC ${ }^{g}$ & 0.58 & $\begin{array}{l}0.54- \\
0.61\end{array}$ & 0.001 & 0.61 & $\begin{array}{l}0.57- \\
0.65\end{array}$ & 0.001 \\
\hline
\end{tabular}

McFadden Pseudo- $\mathrm{R}^{2}=0.026$

\begin{tabular}{|c|c|c|c|c|c|c|c|}
\hline \multirow[t]{3}{*}{$\begin{array}{l}\text { Enabling } \\
\text { Factors }\end{array}$} & NHS onlyc & 0.65 & $\begin{array}{l}0.43- \\
0.98\end{array}$ & 0.042 & 1.34 & $\begin{array}{l}0.81- \\
2.22\end{array}$ & 0.249 \\
\hline & $<4$ Years of Education ${ }^{d}$ & 0.90 & $\begin{array}{l}0.56- \\
1.45\end{array}$ & 0.900 & 1.50 & $\begin{array}{l}0.93- \\
2,43\end{array}$ & 0.096 \\
\hline & Employed $^{\mathrm{e}}$ & 0.55 & $\begin{array}{l}0.31- \\
0.97\end{array}$ & 0.038 & 0.81 & $\begin{array}{l}0.44- \\
1.50\end{array}$ & 0.512 \\
\hline Step 2 & $A_{U C}$ & $\begin{array}{l}0.60(+ \\
0.02)\end{array}$ & $\begin{array}{l}0.56- \\
0.64\end{array}$ & 0.001 & $\begin{array}{l}0.63 \\
(+ \\
0.02)\end{array}$ & $\begin{array}{l}0.59- \\
0.67\end{array}$ & 0.001 \\
\hline
\end{tabular}

McFadden Pseudo- $\mathrm{R}^{2}=0.040$

$(+0.014)$

Need

Variables
Number of

Comorbidities

HRQoL (EQ-5D-3L index $\quad 0.33$ score)

1.12 $\begin{array}{lll}1.03- & 0.011 & 1.22\end{array}$

1.21

$\begin{array}{lll}0.14- & 0.013 & 0.61\end{array}$

0.79 


\begin{tabular}{|c|c|c|c|c|c|c|c|}
\hline & \multirow[b]{2}{*}{$\begin{array}{l}\text { Physical function (HAQ } \\
\text { score) }\end{array}$} & \multicolumn{3}{|c|}{ HighUsers vs. LowUsers } & \multicolumn{3}{|c|}{ GPUsers vs. LowUsers } \\
\hline & & 1.59 & $\begin{array}{l}1.10- \\
2.23\end{array}$ & 0.013 & 1.03 & $\begin{array}{l}0.69- \\
1.53\end{array}$ & 0.902 \\
\hline & Anxiety (HADS-A) & 1.02 & $\begin{array}{l}0.97- \\
1.07\end{array}$ & 0.432 & 1.09 & $\begin{array}{l}1.03- \\
1.14\end{array}$ & 0.002 \\
\hline & $\begin{array}{l}\text { Regular Physical } \\
\text { Exercise }^{f}\end{array}$ & 0.57 & $\begin{array}{l}0.37- \\
0.88\end{array}$ & 0.010 & 0.55 & $\begin{array}{l}0.34- \\
0.89\end{array}$ & 0.014 \\
\hline \multirow[t]{2}{*}{$\begin{array}{l}\text { Step 3: } \\
\text { Final model }\end{array}$} & AUC $^{g}$ & $\begin{array}{l}0.68(+ \\
0.08)\end{array}$ & $\begin{array}{l}0.64- \\
0.71\end{array}$ & 0.001 & $\begin{array}{l}0.69 \\
(+ \\
0.07)\end{array}$ & $\begin{array}{l}0.65- \\
0.73\end{array}$ & 0.001 \\
\hline & $\begin{array}{l}\text { McFadden Pseudo- } R^{2}= \\
0.098(+0.058)\end{array}$ & & & & & & \\
\hline \multicolumn{8}{|c|}{$\begin{array}{l}\text { NHS, National Health System; GP: General Practitioner; HRQoL, Health Related Quality of Life; EQ-5D- } \\
\text { 3L, EuroQol with five dimensions and three levels; HAQ, Health Assessment Questionnaire; HADS-A, } \\
\text { Hospital Anxiety and Depression Scale - Anxiety subscale. Reference Categories: }{ }^{a} \text { Female; }{ }^{b} \text { Lisbon } \\
\text { and Tagus valley; }{ }^{c} \text { Healthcare insurance along with NHS; }{ }^{d} \geq 4 \text { years of education; }{ }^{\text {eNon }} \text { employed or } \\
\text { retired; }{ }^{f} \text { Don't perform regular physical exercise; }{ }^{9} \text { Area Under the ROC Curve }(95 \% \text { CI) }- \text { reference } \\
\text { cluster is LowUsers. Differences in discriminatory capacity (AUC) and in variance of the model } \\
\text { regarding the previous step is shown in brackets. } \chi^{2}(28)=180.328, p<0.001\end{array}$} \\
\hline \multicolumn{8}{|c|}{$\begin{array}{l}\text { This procedure excluded all the participants with missing data. Sample included in the analysis: } \\
\text { Total: } n=838(85,69 \% \text { of the initial sample), HighUsers: } n=295 \text { ( } 86.0 \% \text { of the initial cluster sample); } \\
\text { GPUsers: } n=232(85,29 \% \text { of the initial cluster sample) ; LowUsers: } n=311 \text { ( } 85.67 \% \text { of the initial } \\
\text { cluster sample) }\end{array}$} \\
\hline
\end{tabular}

As seen in the Tables 3 and 4, in the multinomial logistic model, having LowUsers as the reference cluster, the following determinants were associated with HighUsers cluster membership: being younger ( $\mathrm{OR}=$ $0.96,95 \% \mathrm{Cl} 0.95,0.99)$ and reside in Portugal mainland, when compared to reside on islands $(\mathrm{OR}=0.43$, $95 \% \mathrm{Cl} 0.24,0.77)$ as predisposing characteristics; have additional health coverage $(\mathrm{OR}=0.65,95 \% \mathrm{Cl}$ $0.43,0.98)$ and being employed ( $\mathrm{OR}=0.55,95 \% \mathrm{Cl} 0.31-0.97)$ as enabling factors; and higher number of comorbidities $(\mathrm{OR}=1.12,95 \% \mathrm{Cl} 0.31,0.97)$, worse $\mathrm{HRQ}$ oL $(\mathrm{OR}=0.33,95 \% \mathrm{Cl} 0.14,0.79)$, worse physical function $(\mathrm{OR}=1.59,95 \% \mathrm{Cl} 1.10-2.23)$ and no regular physical exercise $(\mathrm{OR}=0.57,95 \% \mathrm{Cl} 0.37,0.88)$ as need variables. The only predisposing characteristic associated with GPUsers membership was geographic location. Residing in the centre when compared to reside in Lisbon region $(O R=2.11,95 \% \mathrm{Cl}$ $1.21,3.68)$, and in Portugal mainland when compared to reside in the Islands region $(\mathrm{OR}=0.42,95 \% \mathrm{Cl}$ $0.21,0.83$ ), increase the probability of being classified as GPUser, with LowUsers as the reference cluster. No enabling factors had statistical association within GPUsers cluster membership. Higher number of comorbidities $(\mathrm{OR}=1.22 .95 \% \mathrm{Cl} 1.11,1.33)$, the presence of anxiety symptoms $(\mathrm{OR}=1.09,95 \% \mathrm{Cl} 1.03$, $1.14)$ and have no regular physical exercise $(\mathrm{OR}=0.5595 \% \mathrm{Cl} 0.34,0.89)$ were the need variables associated with GPU cluster membership. A higher variation in the AUC and in the McFadden pseudo- ${ }^{2}$ occurred when need variables were entered in the model. 
Please place here "Table 3Final Multinomial Regression Model" (presented in the end of the manuscript)

Table 4

Summary of determinants that increase the probability of membership in each healthcare utilisation profile, according to Andersen's Behaviour Model of Healthcare Utilisation

\begin{tabular}{|c|c|c|c|}
\hline & Determinants & HighUsers $^{a}$ & GPUsers ${ }^{a}$ \\
\hline \multirow{3}{*}{$\begin{array}{l}\text { Predisposing } \\
\text { Characteristics }\end{array}$} & Age & - Being Younger & - \\
\hline & $\begin{array}{l}\text { Geographic } \\
\text { Location }\end{array}$ & $\begin{array}{l}\text { - Live in Portugal } \\
\text { Mainland }\end{array}$ & $\begin{array}{l}\text { - Live in Portugal } \\
\text { Mainland }\end{array}$ \\
\hline & & & - Live in the centre region \\
\hline \multirow[t]{2}{*}{ Enabling Factors } & $\begin{array}{l}\text { Healthcare } \\
\text { insurance }\end{array}$ & $\begin{array}{l}\text { - Additional healthcare } \\
\text { coverage }\end{array}$ & - \\
\hline & $\begin{array}{l}\text { Employment } \\
\text { status }\end{array}$ & - Being employed & - \\
\hline \multirow[t]{5}{*}{ Need Variables } & Comorbidities & $\begin{array}{l}\text { - Higher number of } \\
\text { comorbidities }\end{array}$ & $\begin{array}{l}\cdot \text { Higher number of } \\
\text { comorbidities }\end{array}$ \\
\hline & Quality of life & - Worse HRQoL & - \\
\hline & $\begin{array}{l}\text { Physical } \\
\text { function }\end{array}$ & -Worse physical function & - \\
\hline & $\begin{array}{l}\text { Anxiety } \\
\text { symptoms }\end{array}$ & - & - More anxiety symptoms \\
\hline & $\begin{array}{l}\text { Physical } \\
\text { Exercise }\end{array}$ & $\begin{array}{l}\text { - No regular physical } \\
\text { exercise }\end{array}$ & $\begin{array}{l}\text { - No regular physical } \\
\text { exercise }\end{array}$ \\
\hline \multicolumn{3}{|c|}{ aReference Cluster: LowUsers } & \\
\hline
\end{tabular}

\section{Discussion}

\section{Healthcare Services Utilisation in Portugal}

In this study, we identified three profiles of healthcare utilisation according to the services most used by the participants with knee OA. The profile with the highest healthcare utilisation - HighUsers, represents more than $35 \%$ of the sample and was characterised by participants with appointments with the GP, orthopaedic surgeon, physiotherapy sessions and/or with hospitalisation. Given the high number of other medical appointments, this profile is possibly responsible for a high proportion of the total costs spent with people with knee OA in Portugal. As Warwick et al. (2020) concluded, analysing an insurance database with more than 40000 of people with knee OA, the top $30 \%$ of high-payment patients with OA accounted for more than $70 \%$ of overall non-arthroplasty payments (26). 
Primary care is considered the most relevant setting for prevention and management of knee $O A$, where the conservative non-pharmacological interventions should be considered early, and throughout the progression of the disease $(8,9)$. However, in our sample, few participants were enrolled in physiotherapy or regular exercise programmes and a high proportion were overweight. The study of Østeras et al. (2015) found similar data, when analysing a sample of Portuguese people with knee $O A$ in primary healthcare: only $20 \%$ of participants were referred to weight management programmes, and only $43 \%$ were referred to physical exercise programmes, in a similar fashion to other European countries included (27). However, in our sample, the proportion of participants who had undergone physiotherapy treatments (14.4\%) was much lower compared to the $39 \%-52 \%$ observed, for example, in the UK (28). Overall, this data may suggest a weak adoption of the core recommended interventions for the management of knee $O A$, and possibly, be responsible for suboptimal outcomes and higher health costs, in Portugal. Moreover, Bedard et al. (2017) estimated that if health professionals followed current clinical practice guidelines, the noninpatient costs with OA would decrease by $45 \%$ (29). This data should sufficiently alarm health politicians regarding the need for the implementation of effective and recommended modalities in the management of people with knee OA at a national level.

\section{Determinants for Healthcare Services Utilisation}

Overall, the characteristics of our sample are similar to other data related to people with multimorbidity and the older adult population in Portugal, namely given the high proportion of people with lower education, high proportion being overweight or obese, and physically inactive $(5,30)$.

Our findings show that, regardless of clinical need, predisposing characteristics and enabling factors such as age, geographic location, health insurance and employment status, play an important role in healthcare utilisation. This data may disclose that, possibly, the current management of knee OA is heterogenous, not consistent with the needs of the patients, and also, highlights possible inequities in the access of health care (16).

In our analysis, younger and employed participants were positively associated with HighUsers profile. Unlike the data related to general older adults population in Portugal (31), evidence suggests that older adults with knee $O A$ are less likely to be referred to specialised services, like an orthopaedic surgeon, rheumatologist (32) or to physiotherapy (28). Qualitative data suggests that GP's often consider OA as a normal consequence of ageing, attributing low importance to this condition in older adults (33). In contrast, knee $\mathrm{OA}$ is associated with work-related disability, absenteeism, early retirement, psychological distress and low HRQoL in younger patients $(4,34)$. Thus, employed or younger adults with knee OA seem to behave more proactively in seeking help and their physical limitations are generally taken more seriously by GP's, with higher referral rates and consequently, a higher utilisation of healthcare services (33).

Our findings also suggest that geographic location is a determinant to healthcare services utilisation, namely the Islands and Centre region. Both of these regions are far from city centres, with higher proportion of older, less educated and poorer people. These regions experience a shortage of medical 
specialists such as orthopaedic surgeons. Moreover, Madeira and Azores are underserved by primary care units (35). International data suggests that the distance from healthcare units, lack of transport and consequent isolation, and the perception of $\mathrm{OA}$ as self-limited condition, may prevent people from rural areas of seeking healthcare services timely, with lower healthcare resources utilisation as consequence $(36,37)$.

Participants with additional healthcare coverage were more likely to be HighUsers, suggesting that the NHS may not provide optimal access to the appropriate interventions according to the patients' needs, or that the facilitation of access to private sector may enhance the utilisation of healthcare services, regardless of the severity of the disease (38). In accordance with our study, private health insurance was the most frequently cited enabler in Australia for surgical and conservative OA treatments, such as physiotherapy (39).

Overall, our findings suggest that the delivery of healthcare for Portuguese people with knee OA may be inefficient and unfair, where people with better predisposing and enabling features consume a higher amount of healthcare services, than people without those features. Our findings, with the support of the presented literature, should raise concerns regarding the need to tackle health access inequities in Portugal. In this way, the organisation of the health system should guarantee that people with OA receive effective interventions according to clinical severity, and not according to sociodemographic factors.

For predisposing variables, our findings showed that the number of comorbidities is associated with higher healthcare utilisation profiles, mainly with GPUsers profile, as well as anxiety symptoms. People with OA visit primary care mostly in case of multimorbidity (40). However, evidence shows that, in people with $\mathrm{OA}$ and multimorbidity, joint pain is often seen as a low priority problem, brought up late in the consultation, with low referral rates to physiotherapy or specialised care targeted to $O A(40,41)$. This information may explain the stronger association of number of comorbidities with GPUsers profile, than with HighUsers. Regarding anxiety symptoms, contradictory data was found in literature. Anxiety is associated both negatively and positively with the utilisation of healthcare services $(42,43)$. However, it is well known that mental health comorbidities, like anxiety and depression, as well as cardiovascular and metabolic comorbidities are associated with higher severity symptoms and poor outcomes in people with $O A(43,44)$. Thus, the management of people with $O A$, especially with anxiety and/or multimorbidity, should be multidisciplinary personalised and targeted $(8,9)$, which would justify a higher utilisation of healthcare, mostly specialised services, partly in contrast to our data. Thus, we may argue that this subpopulation of patients with knee $\mathrm{OA}$ is undertreated in Portugal, recognising the urge to organise services across healthcare sectors to pursue the delivery of recommended and more effective interventions, mainly to people with poor prognosis.

In our study, physical inactivity was associated with both profiles of higher healthcare utilisation. Sedentary behaviour and being overweight in people with knee $\mathrm{OA}$ is associated with worst physical function, higher risk of cardiovascular comorbidities (7), higher healthcare consumption and higher health-related costs (45). Barriers to physical exercise have been identified in literature that justify the low 
adherence of patients, namely the misbeliefs of health professionals regarding exercise and physiotherapy (46).

As expected, low levels of physical function and HRQoL are associated with HighUsers. A 10-year UK survey reported that disability was the strongest predictor for referral to specialised care and for TKR in people with knee pain (32). Similarly to our data, worse physical function, associated comorbidities, and also radiologic severity were also associated with higher direct and indirect costs in a Spanish survey (47). From another point of view, our data could suggest that people with higher healthcare utilisation in the previous 12 months, do not have better physical function, better quality of life or are undertaking of physical exercise, thus, higher healthcare utilisation does not necessarily reflect better outcomes.

\section{Strengths and Limitations}

This is the first study in Portugal analysing the health services utilisation by people with knee OA at a national level. The large sample, the multi-domains of the dataset and its framing on Andersen's model, provides a comprehensive view of the current healthcare utilisation profiles and its determinants.

Nevertheless, it has some limitations. The cross-sectional design does not does not allow the establishment of a temporal relationship between determinants and healthcare utilisation; thus, cause and effect can be overestimated mainly in modifiable variables like physical function or HRQoL.

Behavioural variables for healthcare utilisation were not controlled. The physical activity variable did not take into account the amount of time spent per week, neither its intensity, thus our results may be, even so, overestimated comparing to the recommendations for physical activity. Public or private appointments, were not distinguished, which could increase the importance of predisposing characteristics and enabling factors in the variance of healthcare utilisation. As self-reported healthcare utilisation relates to the previous 12 months, we acknowledge the possibility of memory bias. The reason for medical appointments or physiotherapy attendance was not controlled, which could increase the association of healthcare insurance with the utilisation of health services. The data used was collected in 2011-2013 but, due to the few specific strategies directed to musculoskeletal diseases in the last decade in Portugal, we cautiously believe that the actual management of OA does not differ from this study.

\section{Implications of the findings}

The results of this study highlight the importance of addressing the inequalities of access and heterogeneity in care, as well as the need to tackle adherence to exercise and enhancement of selfmanagement strategies, e.g., with physiotherapy in primary care, to a much larger proportion of the population with knee OA. A whole system approach needs to consider primary prevention, early detection, cost-effective interventions and appropriate referral, as well as personalised interventions taking into account other comorbidities that are often present in these patients (48). International strategies to address this problem include the implementation of integrated care pathways or models of care, and quality standards to reduce the evidence-practice and evidence-policy gaps (49). These strategies are showing promising results in overall patient satisfaction with care, improvement of patient-level 
outcomes, adherence to guideline recommendations, appropriate use of medication and reduction of absence from work (50).

\section{Conclusion}

We identified three different healthcare services utilisation profiles. The HighUsers profile accounted for more than one third of people with knee OA and it includes GP utilisation, orthopaedic surgeon appointments, physiotherapy and/or hospitalisation. Need variables explained a considerable proportion of the variance in healthcare utilisation, although determinants like younger age and geographic location, having additional healthcare coverage and being employed were associated with higher utilisation of healthcare services. These facts suggest the need for improvement in the access of healthcare services, in the quality of care and, implementation of international recommendations according with clinical severity in people with knee OA.

\section{Abbreviations}

BMI

Body Mass Index

$\mathrm{Cl}$

Confidence Interval

EQ-5D-3L

EuroQol Questionnaire with 5 dimensions and 3 levels

GBP

Gross Domestic Product

GP

General Practitioner

GPUsers

General Practitioner Users profile

HADS-A

Hospital Anxiety and Depression Scale - Anxiety subscale HADS-D

Hospital Anxiety and Depression Scale - Depression subscale

HighUsers

High Healthcare Users profile

HRQoL

Health Related Quality of Life

LowUsers

Low Healthcare Users profile

$\mathrm{NHS}$

National Health Service 
$\mathrm{OA}$

Osteoarthritis

OR

Odds Ratio

TKR

Total Knee Replacement

\section{Declarations}

Funding: This study is funded by national funds through FCT - Fundação para a Ciência e Tecnologia, I. P. under the PhD grant SFRH/BD/148420/2019 awarded to the first author. This protocol was included in the $\mathrm{PhD}$ previously approved project.

EpiReumpaPt was supported by unrestricted grants from Direcção-Geral da Saúde, Fundação Calouste Gulbenkian, Fundação Champalimaud, Fundação AstraZeneca, AbbVie, Merck, Sharp \& Dohme, Pfizer, Roche, Servier, Bial, D3A Medical Systems, Happybrands, Center de Medicina Laboratorial Germano de Sousa, Clínica Médica da Praia da Vitória, CAL-Clínica, Galp Energia, Açoreana Seguros, and individual rheumatologists.

Competing interests: The authors report no conflict of interests.

Acknowledgements: We would like to acknowledge the EpiDoc Unit and EpiReumaPt team for the invaluable assignment of conceptualisation, assembling and to operationalise the main research project - EpireumaPt, and to Deborah Nossiter for her assistance in the writing process of this article.

Data availability statement: The data underlying this article were provided by the EpiDoc Unit - CEDOC by permission. Data will be shared on request to the corresponding author with permission of the EpiDoc Unit group leaders.

Authors contribution: Daniela Costa contributed to the drafting of the manuscript. Daniela Costa, Eduardo B Cruz, Carla Nunes and Ana M Rodrigues contributed to the analysis and interpretation of the data and statistics. Helena Canhão, Jaime Branco and Ana M Rodrigues contributed to the conception and design of the main project (EpiReumaPt), for the provision of study materials, obtaining funding for the main project, administrative/logistic support and in collection of the data. All of the authors critically revised and approved the final manuscript.

\section{References}

1. Safiri S, Kolahi AA, Hoy D, Smith E, Bettampadi D, Mansournia MA, et al. Global, regional and national burden of rheumatoid arthritis 1990-2017: a systematic analysis of the Global Burden of Disease study 2017. Ann Rheum Dis. 2019;78(11):1463. doi:10.1136/annrheumdis-2019-215920. 
2. Salmon JH, Rat AC, Sellam J, Michel M, Eschard JP, Guillemin F, et al. Economic impact of lower-limb osteoarthritis worldwide: a systematic review of cost-of-illness studies. Osteoarthr Cartil. 2016 Sep 1;24(9):1500-8. doi: 10.1016/j.joca.2016.03.012.

3. Branco JC, Rodrigues AM, Gouveia N, Eusebio M, Ramiro S, Machado PM, et al. Prevalence of rheumatic and musculoskeletal diseases and their impact on health-related quality of life, physical function and mental health in Portugal: results from EpiReumaPt- a national health survey. RMD Open. 2016;2(1):e000166. doi:10.1136/rmdopen-2015-000166.

4. Laires PA, Canhao H, Rodrigues AM, Eusebio M, Gouveia M, Branco JC. The impact of osteoarthritis on early exit from work: results from a population-based study. BMC Public Health. 2018;18(1):472. doi:10.1186/s12889-018-5381-1.

5. Ministério da Saúde. Retrato da Saúde. Portugal; 2018.

6. Turkiewicz A, Petersson IF, Björk J, Hawker G, Dahlberg LE, Lohmander LS, et al. Current and future impact of osteoarthritis on health care: A population-based study with projections to year 2032. Osteoarthr Cartil. 2014;22(11):1826-32.

7. Hawker G. Osteoarthritis is a serious disease. Clin Exp Rheumatol [Internet]. 2019;37(120).

8. Bannuru RR, Osani MC, Vaysbrot EE, Arden NK, Bennell K, Bierma-Zeinstra SMA, et al. OARSI guidelines for the non-surgical management of knee, hip, and polyarticular osteoarthritis. Osteoarthr Cartil. 2019;27(11):1578-89. doi:10.1016/j.joca.2019.06.011.

9. Kolasinski SL, Neogi T, Hochberg MC, Oatis C, Guyatt G, Block J, et al. 2019 American College of Rheumatology/Arthritis Foundation Guideline for the Management of Osteoarthritis of the Hand, Hip, and Knee. Arthritis Rheumatol. 2020. doi:10.1002/art.41142.

10. Price AJ, Alvand A, Troelsen A, Katz JN, Hooper G, Gray A, et al. Knee replacement. Lancet. 2018;392(10158):1672-82. doi:10.1016/S0140-6736(18)32344-4.

11. Hagen KB, Smedslund G, Osteras N, Jamtvedt G. Quality of Community-Based Osteoarthritis Care: A Systematic Review and Meta-Analysis. Arthritis Care Res. 2016;68(10):1443-52. doi:10.1002/acr.22891.

12. Kingsbury SR, Gross HJ, Isherwood G, Conaghan PG. Osteoarthritis in Europe: impact on health status, work productivity and use of pharmacotherapies in five European countries. Rheumatol. 2014;53(5):937-47. doi:10.1093/rheumatology/ket463.

13. Pabinger $\mathrm{C}$, Lothaller $\mathrm{H}$, Geissler $\mathrm{A}$. Utilization rates of knee-arthroplasty in OECD countries. Osteoarthr Cartil. 2015;23(10):1664-73. doi:10.1016/j.joca.2015.05.008.

14. Chen F, Su W, Bedenbaugh AV, Oruc A, Chen F, Su W, et al. Health care resource utilization and burden of disease in a U. S. Medicare population with a principal diagnosis of osteoarthritis of the knee. $\mathrm{J}$ Med Econ. 2020;23(10):1151-8. doi:10.1080/13696998.2020.1801453.

15. Lo TKT, Parkinson L, Cunich M, Byles J. Factors associated with the health care cost in older Australian women with arthritis: an application of the Andersen 's Behavioural Model of Health Services Use. Public Health. 2015;1-8. 
16. Babitsch B, Gohl D, von Lengerke T. Re-revisiting Andersen's Behavioral Model of Health Services Use: A Systematic Review of Studies From 1998-2011. Psychosoc Med. 2012;11.

17. Simões J, Augusto GF, Fronteira I, Hernández-Quevedo C. Portugal: Health system review. Health Syst Transit. 2017;19(2).

18. Rodrigues Am Fau - Gouveia N. Gouveia N Fau - da Costa LP, da Costa Lp Fau - Eusebio M, Eusebio M, Fau - Ramiro S, Ramiro S, Fau - Machado P, Machado P, Fau - Mourao AF, et al. EpiReumaPt- the study of rheumatic and musculoskeletal diseases in Portugal: a detailed view of the methodology. Acta Reum Port. 2015;40(2).

19. Altman R, Asch E, Bloch D, Bole G, Borenstein D, Brandt K, et al. Development of criteria for the classification and reporting of osteoarthritis. Classification of osteoarthritis of the knee. Diagnostic and Therapeutic Criteria Committee of the American Rheumatism Association. Arthritis Rheum. 1986;29(8):1039-49.

20. Ferreira LN, PL N, Oppe M, Qual Life R. The valuation of the EQ-5D in Portugal. Qual Life Res [Internet]. 2014;23(2).

21. Pais-Ribeiro J, Silva I, Ferreira T, Martins A, Meneses R, Baltar M. Validation study of a Portuguese version of the Hospital Anxiety and Depression Scale. Psychol Heal Med. 2007;12(2):225-7. doi:10.1080/13548500500524088.

22. Fries JF, Spitz P, Kraines RG, Holman HR. Measurement of patient outcome in arthritis. Arthritis Rheum. 1980;23(2):137-45.

23. Blacketer C, Gill T, Taylor A, Hill C. Prevalence and healthcare usage of knee pain in South Australia: a population-based study. Intern Med J. 2019;49(9):1105-10. doi:10.1111/imj.14237.

24. Kent $P$, Jensen RK, Kongsted $A$. A comparison of three clustering methods for finding subgroups in MRI, SMS or clinical data: SPSS TwoStep Cluster analysis, Latent Gold and SNOB. BMC Med Res Methodol. 2014;14(1):1-14.

25. Marôco J. Análise Estatística com o SPSS Statistics. 7th Editio. ReportNumber; 2018.

26. Warwick H, O'Donnell J, Mather RC 3rd, Jiranek W. Disparity of health services in patients with knee osteoarthritis before total knee arthroplasty. Arthroplast Today. 2020;6(1):81-7. doi:10.1016/j.artd.2019.11.008.

27. Osteras N, Jordan KP, Clausen B, Cordeiro C, Dziedzic K, Edwards J, et al. Self-reported quality care for knee osteoarthritis: comparisons across Denmark, Norway, Portugal and the UK. RMD Open. 2015;1(1):e000136. doi:10.1136/rmdopen-2015-000136.

28. Smith T, Collier TS, Smith B, Mansfield M. Who seeks physiotherapy or exercise treatment for hip and knee osteoarthritis? A cross-sectional analysis of the English Longitudinal Study of Ageing. Int $\mathrm{J}$ Rheum Dis. 2019;22(5):897-904. doi:10.1111/1756-185X.13480.

29. Bedard NA, Dowdle SB, Anthony CA, DeMik DE, McHugh MA, Bozic KJ, et al. The AAHKS Clinical Research Award: What Are the Costs of Knee Osteoarthritis in the Year Prior to Total Knee Arthroplasty? J Arthroplasty. 2017;32(9):8-S. 10.e1. doi: 10.1016/j.arth.2017.01.011. 
30. Prazeres F, Santiago L. Prevalence of multimorbidity in the adult population attending primary care in Portugal: a cross-sectional study. BMJ Open. 2015;5(9):e009287. doi:10.1136/bmjopen-2015009287.

31. Rodrigues AM, Gregorio MJ, Sousa RD, Dias SS, Santos MJ, Mendes JM, et al. Challenges of Ageing in Portugal: Data from the EpiDoC Cohort. Acta Med Port. 2018;31(2):80-93. doi:10.20344/amp.9817.

32. Jinks C, Vohora K, Young J, Handy J, Porcheret M, Jordan KP. Inequalities in primary care management of knee pain and disability in older adults: an observational cohort study. Rheumatol. 2011;50(10):1869-78. doi:10.1093/rheumatology/ker179.

33. Wallis JA, Taylor NF, Bunzli S, Shields N. Experience of living with knee osteoarthritis: a systematic review of qualitative studies. BMJ Open. 2019;9(9):e030060. doi:10.1136/bmjopen-2019-030060.

34. Ackerman IN, Bucknill A, Page RS, Broughton NS, Roberts C, Cavka B, et al. The substantial personal burden experienced by younger people with hip or knee osteoarthritis. Osteoarthr Cartil. 2015;23(8):1276-84. doi:10.1016/j.joca.2015.04.008.

35. OECD, European Observatory on Health, Policies. Portugal: Country Health Profile 2019. 2019. doi: https://doi.org/10.1787/85ed94fc-en.

36. Brundisini F, Giacomini M, DeJean D, Vanstone M, Winsor S, A. S. Chronic disease patients' experiences with accessing health care in rural and remote areas: a systematic review and qualitative meta-synthesis. Ont Heal Technol Assess Ser. 2013;13(15).

37. Hollick RJ, Macfarlane GJ. Do patients with rheumatic diseases living in rural settings have poorer outcomes? Results from a systematic review of the literature. Arthritis Care Res [Internet]. 2020;10.

38. Folland S, Goodman A, Stano M. The Economics of Health and Health Care. New Jersey: Pearson Education, Inc; 2013.

39. Ackerman IN, Livingston JA, Osborne RH. Personal Perspectives on Enablers and Barriers to Accessing Care for Hip and Knee Osteoarthritis. Phys Ther. 2016;96(1):26-36. doi:10.2522/ptj.20140357.

40. Prazeres F, Santiago L. Prevalence of multimorbidity in the adult population attending primary care in Portugal: a cross-sectional study. 2015;5:9287. doi: 10.1136/bmjopen-2015.

41. Paskins Z, Sanders T, Croft PR, Hassell AB. The Identity Crisis of Osteoarthritis in General Practice: A Qualitative Study Using Video-Stimulated Recall. Ann Fam Med. 2015;13(6):537-44. doi:10.1370/afm.1866.

42. Thorstensson CA, Gooberman-Hill R, Adamson J, Williams S, Dieppe P. Help-seeking behaviour among people living with chronic hip or knee pain in the community. BMC Musculoskelet Disord. 2009;10(1):153. doi:10.1186/1471-2474-10-153.

43. Sharma A, Kudesia P, Shi Q, Gandhi R. Anxiety and depression in patients with osteoarthritis: Impact and management challenges. Open Access Rheumatol Res Rev. 2016;8:103-13.

44. Parkinson L, Waters DL, Franck L. Systematic review of the impact of osteoarthritis on health outcomes for comorbid disease in older people. Osteoarthr Cartil. 2017;25(11):1751-70. 
doi:10.1016/j.joca.2017.07.008.

45. Johnston SS, Ammann E, Scamuffa R, Samuels J, Stokes A, Fegelman E, et al. Association of body mass index and osteoarthritis with healthcare expenditures and utilization. Obes Sci Pr. 2020;6(2):139-51. doi:10.1002/osp4.398.

46. Cottrell E, Roddy E, Foster NE. The attitudes, beliefs and behaviours of GPs regarding exercise for chronic knee pain: a systematic review. BMC Fam Pract. 2010;11(1):4. doi:10.1186/1471-2296-11-4.

47. Loza E, Lopez-Gomez JM, Abasolo L, Maese J, Carmona L, Batlle-Gualda E, et al. Economic burden of knee and hip osteoarthritis in Spain. Arthritis Rheum. 2009;61(2):158-65. doi:10.1002/art.24214.

48. Callahan L, Ambrose K, Albright A, Altpeter M, Golightly Y, Huffman K, et al. Public Health Interventions for Osteoarthritis - updates on the Osteoarthritis Action Alliance's efforts to address the 2010 OA Public Health Agenda Recommendations. Clin Exp Rheumatol [Internet]. 2019;37(120).

49. Speerin R, Slater H, Li L, Moore K, Chan M, Dreinhofer K, et al. Moving from evidence to practice: Models of care for the prevention and management of musculoskeletal conditions. Best Pr Res Clin Rheumatol. 2014;28(3):479-515. doi:10.1016/j.berh.2014.07.001.

50. Allen KD, Choong PF, Davis AM, Dowsey MM, Dziedzic KS, Emery C, et al. Osteoarthritis: Models for appropriate care across the disease continuum. Best Pr Res Clin Rheumatol. 2016;30(3):503-35. doi:10.1016/j.berh.2016.09.003.

\section{Figures}




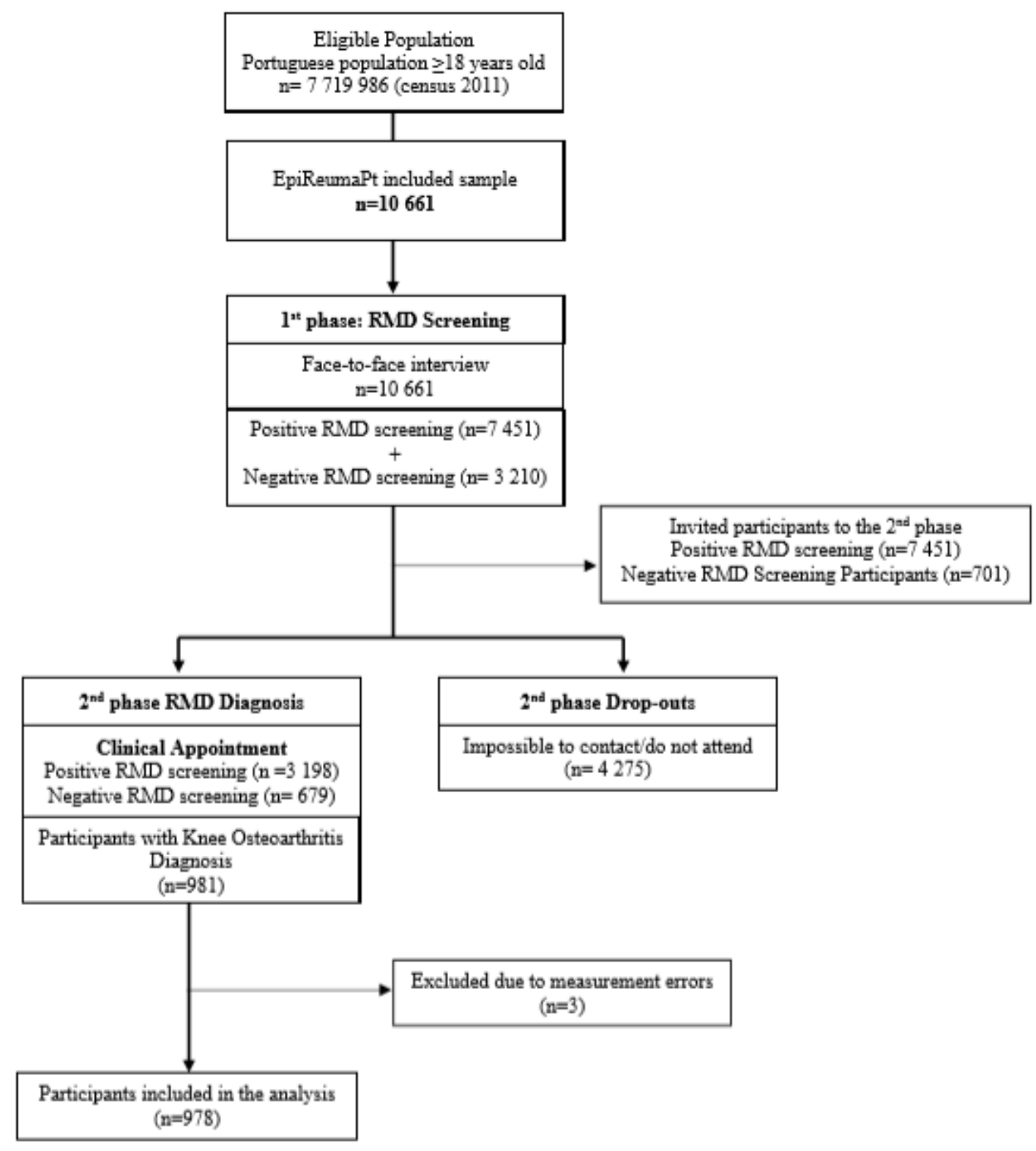

\section{Figure 1}

Flowchart of EpiReumaPt design. RMD, Rheumatic and Musculoskeletal Diseases

\section{Supplementary Files}

This is a list of supplementary files associated with this preprint. Click to download.

- TableSupplement.docx 